Annales UMCS

Informatica

Lublin-Polonia

Sectio AI

http://www.annales.umcs.lublin.pl/

\title{
On departure process in the batch arrival queue with single vacation and setup time
}

\author{
Wojciech M. Kempa* \\ Institute of Mathematics, Silesian University of Technology, \\ ul. Kaszubska 23, Gliwice, Poland.
}

\begin{abstract}
A single-server queueing system of $M^{X} / G / 1$ type with unlimited buffer size is considered. Whenever the system becomes empty, the server takes a single compulsory vacation that is independent of the arrival process. The service of the first customer after the vacation is preceded by a random setup time. We distinguish two cases of the evolution of the system: when the setup time begins after the vacation only, or if it begins at once when the first group of customers enters. In the paper we investigate the departure process $h(t)$ that at any fixed moment $t$ takes on a random value equal to the number of customers completely served before $t$. An explicit representation for Laplace Transform of probability generating function of departure process is derived and written down by means of transforms of four crucial "input" distributions of the system and factors of a certain factorization identity connected with them. The results are obtained using the method consisting of two main stages: first we study departure process on a single vacation cycle for an auxiliary system and direct the analysis to the case of the system without vacations, applying the formula of total probability; next we use the renewal-theory approach to obtain a general formula.
\end{abstract}

\section{Introduction}

In the article we deal with a batch arrival queueing system with exponentially distributed interarrival times, generally distributed individual service times and infinite buffer size (the system of $M^{X} / G / 1 / \infty$ type, using the Kendall's notation). The system

*wojciech.kempa@polsl.pl 
starts working in the so called "standard" regime i.e. it is empty before initializing and the first group of customers enters at time $t=0$. Every time when the system empties, it takes a single generally distributed vacation time during which the server is turned off. The first service after finishing the vacation is preceded by a setup time of random length. In fact, we distinguish two types of possible evolutions of the system:

(A) The case of "exhaustive vacation", in that the setup time begins only when the vacation finishes, even then there are some arrivals during the vacation;

(B) The case of "non-exhaustive vacation", in that if the first arrival occurs during the vacation, the setup time begins at this moment (the vacation lasts until the first group of customers arrives).

If the system is still empty after finishing the vacation, the server does not take another vacation but waits for customers. When the first arrival occurs, the setup time begins immediately.

In the paper we investigate the departure process $h(t)$ of the system (in other words: the process counting the served customers) that at any fixed moment $t$ takes on a random value equal to the number of customers completely served before $t$.

Systems with server vacations can be good models for different situations occurring in telecommunication, manufacturing and computer science. A queueing system with a temporary unavailable server can describe the service process of applicants at the cash desk in that the break is ordered when the queue empties. In computer science, the vacation time can be treated as a time for server's maintenance etc.

Of special significance are the systems with batch arrivals of customers which can be good models of evolution of the Internet traffic. The results obtained for vacation models concern mainly the stationary characteristics of the system. But in practice, the convergence rate of transient characteristics to the stationary ones is so slow that the equilibrium state does not convey the real state of the system.

The overview of early results concerning the vacation models can be found in Doshi [5]. The monograph by Takagi [12] comprises the in-depth analysis of the $M / G / 1$ queues with vacations. In Tian and Zhang [14] one can find the results for models with different types of vacation policies. The case of exponentially distributed batch arrivals is studied in Choudhury [3] and Choudhury and Kalita [4]. In Baba [1] the $M^{X} / G / 1$ vacation model is studied using the method of supplementary variables. The fundamental result for queueing systems with vacations (the so called decomposition property) was stated originally by Fuhrmann and Cooper [6] in 1985 for the systems of $M / G / 1$ type. They proved that the stationary queue size in the system is a sum of two or more independent random variables, one of them is the stationary queue size in the correspondent system without vacations.

A batch arrival queueing system of $G I^{X} / G / 1$ type with single vacations was studied by Kempa in [10]. The explicit representation for the joint transform of the first busy period, the first vacation time and the number of customers served during the first busy period was obtained for the case of exponentially distributed single vacation time. 
The departure process for the batch arrival system of general type (without vacations) was studied in Kempa [9] where the representation for its double transform was derived. Some results for this characteristic in the system with single vacations were obtained in Kempa [11]. In Tang [13] some results for departure process in the $M^{X} / G / 1$ system with single vacations are derived in the transient case.

In the present article we study departure process in the single vacation system with setup times. The setup time can be interpreted as a time needed for the system to reach the full efficiency in service. The case (B) of the system was investigated for example by Choudhury in [2] where decomposition results for the steady-state queue size distributions were obtained.

We will obtain results for departure process in the single vacation system with setup time using the procedure that consists of a few steps. First, we will consider a certain simplified system which starts working with a vacation time and waits for customers (we call this system a "waiting" one). For such a system we will find representations for the probability generating functions (PGF) of the Laplace Transforms (LT) of departure processes for the variants (A) and (B) separately. Using the formula of total probability we will write the equations that connect departure processes in the "waiting" system with those in the corresponding system without vacations. Next, considering the renewal process determined by successive vacation cycles, we will obtain the results for the original system in cases (A) and (B).

Thus, the paper is organized as follows. In the next Section 2 we will give the formal description of the system. Section 3 presents the auxiliary results obtained for the corresponding system without vacations. In Section 4 we present the results for the departure process in the auxiliary system with vacation and setup time. The last Section 5 generalizes the results from Section 4 .

\section{The formal description of the system}

We will analyze commonly the vacation model (in variants (A) and (B)) and the corresponding system without vacations. All essential distributions for the systems with and without vacations are assumed to be the same. Let $\alpha^{-1}$ be the mean interarrival time and $F(\cdot)$ and $f(\cdot)$ denote the distribution function (DF) and Laplace-Stieltjes Transform (LST) of service time respectively. The arriving batch of customers has size $k$ with probability $p_{k}$, where $\sum_{k=1}^{\infty} p_{k}=1$. Let $p(z)=\sum_{k=1}^{\infty} p_{k} z^{k},|z| \leq 1$ be the PGF of the sequence $\left(p_{k}\right)$. Besides, let $p_{n}^{k *}$ and $F^{k *}(\cdot)$ be the $k$-fold convolutions of appropriate distributions.

The evolution of the system with vacations, working in the "standard" regime, can be considered wit the successive vacation cycles $c_{0}, c_{1}, c_{2}$ etc. which finish every time when the system is free of customers. The memoryless property of exponential distribution ensures that $c_{i}$ are independent random variables and have for $i=1,2, \ldots$ the same DFs. Let $G_{0}(\cdot)$ and $G_{\gamma}(\cdot), \gamma=A, B$ be DFs of $c_{0}$ and $c_{i}, i \geq 1$ (in the cases of variants 
(A) and (B)) respectively. Denote by $g_{0}(\cdot)$ and $g_{\gamma}(\cdot)$ LSTs of DFs $G_{0}(\cdot)$ and $G_{\gamma}(\cdot)$ respectively. Vacation cycles in the case (A) can be formally decomposed as follows

$$
c_{i}=v_{i}+\delta_{i}+u_{i}+\tau_{i}, \quad i \geq 0,
$$

where $v_{i}$ is the $i$ th vacation, $\delta_{i}$ denotes the $i$ th idle period, $u_{i}$ is the $i$ th setup time and $\tau_{i}$ represents the $i$ th busy period of the system. Of course, $v_{0}=\delta_{0}=u_{0}=0$ and $\delta_{i}$ can be 0 (for $i \geq 1$ ) if the first arrival occurs during $v_{i}$. Denote by $V(\cdot)$ and $U(\cdot)$ DFs of random variables $v_{i}$ and $u_{i}$ respectively. Let $v(\cdot)$ and $u(\cdot)$ be LSTs of DFs $V(\cdot)$ and $U(\cdot)$ respectively.

Similarly, in the case (B) we obtain:

$$
c_{0}=\tau_{0}, \quad c_{i}=\xi+u_{i}+\tau_{i}, \quad i \geq 1,
$$

where $\xi$ is the exponentially distributed random variable (with the mean $\alpha^{-1}$ ) and can be interpreted as a time from the beginning of $c_{i}$ to the first arrival.

\section{$3 \quad$ Auxiliary results}

Let us consider the $M^{X} / G / 1$ system without vacations and setup times corresponding to the original one (we will call this system the "ordinary" one). Let us introduce two different initial conditions for such a system:

- the system is empty before initializing, the first arrival occurs at the time $t=0$, and at $t=0+$ we have exactly $n$ customers present in the system (we denote all probabilities and expected values under such a condition by $\mathbf{P}_{\mathrm{n}}\{\cdot\}$ and $\mathbf{E}_{\mathrm{n}}\{\cdot\}$ respectively);

- the system starts working in the "standard" regime (we use the notation $\mathbf{P}_{\text {std }}\{\cdot\}$ and $\mathbf{E}_{\text {std }}\{\cdot\}$ for such a condition).

Denote by $\widehat{\tau}_{1}$ the first busy period of the system, by $\widehat{\delta}_{1}$ the first idle time and by $N\left(\widehat{\tau}_{1}\right)$ the number of customers completely served during $\widehat{\tau}_{1}$ for the "ordinary" system.

All theorems below were originally proved for the $G I^{X} / G / 1$ queueing system. We state the versions for the $M^{X} / G / 1$ system, saving the notations introduced in Section 2 .

In Bratiichuk and Kempa [7] one can find the following two theorems (the second one we cite in a slightly different form):

Theorem 1. For any $\lambda \geq 0$ and $|\theta| \leq 1$ we have:

$$
\mathbf{E}_{\text {std }}\left\{e^{-\lambda \widehat{T}_{1}} \theta^{N\left(\widehat{\tau}_{1}\right)}\right\}=1-f_{+}(\theta, \lambda, 0, \alpha) .
$$


Theorem 2. For any $\lambda \geq 0,|\theta| \leq 1$ and $n \geq 1$ we have:

$$
\begin{gathered}
\mathbf{E}_{\mathrm{n}}\left\{e^{-\lambda \widehat{\tau}_{1}} \theta^{N\left(\widehat{\tau}_{1}\right)}\right\}= \\
=\theta^{n+1} \int_{0}^{\infty} e^{-\lambda y} \int_{-0}^{y} Q(\theta, \lambda, y-v, \alpha) d P_{+}^{(0)}(\theta, \lambda, v, \alpha) d F^{n *}(y),
\end{gathered}
$$

where

$$
Q(\theta, \lambda, x, \alpha)=\int_{x}^{\infty} d F_{+}(\theta, \lambda, y, \alpha)-f_{+}(\theta, \lambda, 0) e^{-\alpha x}
$$

In the above theorems the functions $P_{+}^{(0)}, F_{+}$and $f_{+}$are connected with the factorization identity (see Bratiichuk and Kempa [7] for more details):

$$
1-\frac{\alpha p(\theta f(\lambda-s))}{s+\alpha}=f_{+}(\theta, \lambda, s, \alpha) f_{-}(\theta, \lambda, s, \alpha)
$$

where $\operatorname{Re}(s) \in[0, \lambda],|\theta| \leq 1$. Besides

$$
\begin{gathered}
f_{+}(\theta, \lambda, s, \alpha)=1+\int_{0}^{\infty} e^{-s x} d F_{+}(\theta, \lambda, x, \alpha), \\
\frac{1}{f_{+}(\theta, \lambda, s, \alpha)}=1+\int_{0}^{\infty} e^{-s x} d P_{+}(\theta, \lambda, x, \alpha)
\end{gathered}
$$

and

$$
P_{+}^{(0)}(\theta, \lambda, x, \alpha)=I\{x>0\}+P_{+}(\theta, \lambda, x, \alpha) .
$$

Let $h(t)$ be the number of customers completely served before $t$ (departure process). Introduce the following notations:

$$
\begin{gathered}
\widehat{D}_{\mathrm{n}}(\theta, \lambda, \alpha)=\sum_{m=0}^{\infty} \theta^{m} \int_{0}^{\infty} e^{-\lambda t} \mathbf{P}_{\mathrm{n}}\left\{h(t)=m, t \in \widehat{\tau}_{1}\right\} d t, \\
\widehat{D}_{\mathrm{std}}(\theta, \lambda, \alpha)=\sum_{m=0}^{\infty} \theta^{m} \int_{0}^{\infty} e^{-\lambda t} \mathbf{P}_{\mathrm{std}}\left\{h(t)=m, t \in \widehat{\tau}_{1}\right\} d t,
\end{gathered}
$$

where $\lambda>0$ and $|\theta| \leq 1$.

The following theorem is proved in Kempa [11] (we state the version for the $M^{X} / G / 1$ system): 
Theorem 3. For any $\lambda>0$ and $|\theta| \leq 1$ the following formula is true:

$$
\begin{gathered}
\widehat{D}_{\mathrm{n}}(\theta, \lambda, \alpha)=\frac{\theta(1-f(\lambda))}{\lambda(1-\theta f(\lambda))}\left[1-(\theta f(\lambda))^{n}\right. \\
\left.+\theta^{n} f_{+}(\theta, \lambda, 0, \alpha) \int_{0}^{\infty} e^{-\lambda y} \int_{0-}^{y}\left(1-e^{-\alpha(y-v)}\right) d P_{+}^{(0)}(\theta, \lambda, v, \alpha) d F^{n *}(y)\right] .
\end{gathered}
$$

Since

$$
\mathbf{P}_{\text {std }}\{\cdot\}=\sum_{n=1}^{\infty} p_{n} \mathbf{P}_{\mathrm{n}}\{\cdot\},
$$

then from the above theorem follows

Corollary 1. For any $\lambda>0$ and $|\theta| \leq 1$ the following equality holds:

$$
\begin{gathered}
\widehat{D}_{\text {std }}(\theta, \lambda, \alpha)=\frac{\theta(1-f(\lambda))}{\lambda(1-\theta f(\lambda))}\left[1-p(\theta f(\lambda))+f_{+}(\theta, \lambda, 0, \alpha)\right. \\
\left.\times \int_{0}^{\infty} e^{-\lambda y} \int_{0-}^{y}\left(1-e^{-\alpha(y-v)}\right) d P_{+}^{(0)}(\theta, \lambda, v, \alpha) \sum_{n=1}^{\infty} p_{n} \theta^{n} d F^{n *}(y)\right] .
\end{gathered}
$$

\section{Departure process in the "waiting" system}

Let us consider the "waiting" system of $M^{X} / G / 1$ type on its first vacation cycle $c_{1}$ (in the "waiting" system we have $c_{0}=0$ ). All probabilities for the "waiting" system will be denoted by $\mathbf{P}_{\mathrm{w}(\mathrm{A})}\{\cdot\}$ or $\mathbf{P}_{\mathrm{w}(\mathrm{B})}\{\cdot\}$ depending on the variant of the original system. It is possible, applying the formula of total probability, to express departure process in such a system using the same characteristic in the correspondent system without vacations and setup times ("ordinary") on its first busy period. Indeed, in the case of evolution (A) we get

$$
\begin{gathered}
\mathbf{P}_{\mathrm{w}(\mathrm{A})}\left\{h(t)=m, t \in c_{1}\right\}=I\{m=0\}\left[e^{-\alpha t}+\left(1-e^{-\alpha t}\right)(1-V(t))\right. \\
\left.+\alpha \int_{0}^{t} e^{-\alpha x} V(x)(1-U(t-x)) d x+\int_{0}^{t}\left(1-e^{-\alpha y}\right)(1-U(t-y)) d V(y)\right] \\
+\sum_{i=0}^{\infty} \sum_{n=i+1}^{\infty} p_{n}^{(i+1) *} \int_{0}^{t} d V(y)\left[\alpha \int_{0}^{y} e^{-\alpha x} d x \int_{0}^{t-y} \frac{(\alpha(z+y-x))^{i} e^{-\alpha(z+y-x)}}{i !}\right. \\
\times \mathbf{P}_{\mathrm{n}}\left\{h(t-y-z)=m, t-y-z \in \widehat{\tau}_{1}\right\} d U(z) \\
\left.+\alpha \int_{y}^{t} e^{-\alpha x} d x \int_{0}^{t-x} \frac{(\alpha z)^{i} e^{-\alpha z}}{i !} \mathbf{P}_{\mathrm{n}}\left\{h(t-z-x)=m, t-z-x \in \widehat{\tau}_{1}\right\} d U(z)\right] .
\end{gathered}
$$


Let us briefly comment the right side of (15). The first departure occurs after $t$ (thus $m=0)$ definitely in the following situations:

- the first arrival occurs after $t$ (the first summand);

- the first arrival occurs before $t$ but the vacation time ends after $t$ (the second summand);

- the first arrival occurs before $t$ and after the vacation but the setup time ends after $t$ (the third summand);

- the first arrival occurs inside the vacation which ends before $t$ but the setup time ends after $t$ (the fourth summand).

Two last summands on the right side of (15) present the situation in that the setup time ends before $t$ and hence, beginning with this moment, we can describe the evolution of the "waiting" system using the evolution of the "ordinary" one (without vacation and setup time) with a fixed number of customers at the initial moment, on its first busy period $\widehat{\tau}_{1}$.

In the case of evolution (B) we have

$$
\begin{aligned}
\mathbf{P}_{\mathrm{w}(\mathrm{B})}\{h(t)= & \left.m, t \in c_{1}\right\}=I\{m=0\}\left[e^{-\alpha t}+\alpha \int_{0}^{t}(1-U(t-x)) e^{-\alpha x} d x\right] \\
& +\alpha \sum_{i=0}^{\infty} \sum_{n=i+1}^{\infty} p_{n}^{(i+1) *} \int_{0}^{t} e^{-\alpha x} \int_{0}^{t-x} \frac{(\alpha y)^{i}}{i !} e^{-\alpha y} \\
& \times \mathbf{P}_{\mathrm{n}}\left\{h(t-x-y)=m, t-x-y \in \widehat{\tau}_{1}\right\} d U(y) d x .
\end{aligned}
$$

The first summand presents the situation when we have definitely no departure before $t$ : the first arrival occurs after $t$ or the setup time ends after $t$. The second (last) summand on the right side of (16) describes the situation in that the setup time ends before $t$ thus we can introduce the departure process in the "ordinary" system. As one can expect, the right side in (16) does not depend on the d.f. $V(\cdot)$ of vacation time.

Now, for $\lambda>0$ and $|\theta| \leq 1$ we obtain from (15)

$$
\begin{gathered}
D_{\mathrm{A}}(\theta, \lambda, \alpha)=\sum_{m=0}^{\infty} \theta^{m} \int_{0}^{\infty} e^{-\lambda t} \mathbf{P}_{\mathrm{w}(\mathrm{A})}\left\{h(t)=m, t \in c_{1}\right\} d t= \\
=\frac{\alpha}{\alpha+\lambda}+\frac{[1-\lambda-u(\lambda)][(\alpha+\lambda) v(\lambda)-\lambda v(\alpha+\lambda)]}{\lambda(\alpha+\lambda)} \\
+\sum_{m=0}^{\infty} \theta^{m} \sum_{i=0}^{\infty} \sum_{n=i+1}^{\infty} \frac{\alpha^{i+1} p_{n}^{(i+1) *}}{(i+1) !} \widehat{D}_{\mathrm{n}}(\theta, \lambda, \alpha)\left[\int_{0}^{\infty} e^{-(\alpha+\lambda) y} d V(y)\right. \\
\left.\times \int_{0}^{\infty} e^{-(\alpha+\lambda) z}\left[(z+y)^{i+1}-z^{i+1}\right] d U(z)+\frac{v(\alpha+\lambda)}{\alpha+\lambda} \int_{0}^{\infty} e^{-(\alpha+\lambda) z} z^{i} d U(z)\right] .
\end{gathered}
$$

Similarly, from (16) we get

$$
\begin{gathered}
D_{\mathrm{B}}(\theta, \lambda, \alpha) \sum_{m=0}^{\infty} \theta^{m} \int_{0}^{\infty} e^{-\lambda t} \mathbf{P}_{\mathrm{w}(\mathrm{B})}\left\{h(t)=m, t \in c_{1}\right\} d t=\frac{1}{\alpha+\lambda}+\frac{\alpha(1-u(\lambda))}{\lambda(\alpha+\lambda)} \\
+\frac{1}{\alpha+\lambda} \widehat{D}_{\mathrm{n}}(\theta, \lambda, \alpha) \sum_{i=0}^{\infty} \sum_{n=i+1}^{\infty} \frac{\alpha^{i+1} p_{n}^{(i+1) *}}{i !} \int_{0}^{\infty} e^{-(\alpha+\lambda) y} y^{i} d U(y) .
\end{gathered}
$$




\section{General results}

Let us return to the original system with single vacations and setup times. We have

$$
\mathbf{P}\{h(t)=m\}=\sum_{k=0}^{\infty} \mathbf{P}\left\{h(t)=m, t \in c_{k}\right\} .
$$

Let us denote by $\widehat{q}(n)$ the probability that exactly $n$ customers are served during $c_{0}$. Of course, this probability is the same for the variants $(\mathbf{A})$ and $(\mathbf{B})$ of the evolution of the system. Besides, let $q_{\mathrm{A}}(n)$ and $q_{\mathrm{B}}(n)$ be probabilities that $n$ customers are completely served during $c_{i}, i=1,2, \ldots$ in the cases of evolution $(\mathbf{A})$ and $(\mathbf{B})$ respectively i.e.

$$
q_{\gamma}(n)=\mathbf{P}\left\{h\left(T_{k+1}^{\gamma}\right)-h\left(T_{k}^{\gamma}\right)=n\right\}, \quad k \geq 1, \quad \gamma=A, B,
$$

where $T_{k}^{\gamma}$ is the moment of the beginning of the $k$ th vacation cycle $c_{k}$ in the variant $\gamma$ of the evolution of the system. Let us put for $|\theta| \leq 1$ and $\gamma=A, B$

$$
\widehat{q}(\theta)=\sum_{n=1}^{\infty} \widehat{q}(n) \theta^{n}, \quad q_{\gamma}(\theta)=\sum_{n=1}^{\infty} q_{\gamma}(n) \theta^{n} .
$$

Of course, for $\gamma=A, B$ we have

$$
\mathbf{P}_{\gamma}\left\{h(t)=m, t \in c_{0}\right\}=\mathbf{P}_{\text {std }}\left\{h(t)=m, t \in \widehat{\tau}_{1}\right\}
$$

The formula of total probability leads to

$$
\begin{gathered}
g_{\mathrm{A}}(\lambda)=\mathbf{E}_{\mathrm{A}}\left\{e^{-\lambda c_{1}}\right\}=\alpha \sum_{i=0}^{\infty} \sum_{n=i+1}^{\infty} p_{n}^{(i+1) *} \mathbf{E}_{\mathrm{n}}\left\{e^{-\lambda \widehat{\tau}_{1}}\right\} \\
\times\left[\int_{0}^{\infty} e^{-\alpha x} d x \int_{x}^{\infty} e^{-\lambda y} d V(y) \int_{0}^{\infty} e^{-\lambda z} \frac{(\alpha(z+y-x))^{i} e^{-\alpha(z+y-x)}}{i !} d U(z)\right. \\
+\frac{v(\alpha+\lambda)}{\alpha+\lambda} \int_{0}^{\infty} e^{\left.-(\alpha+\lambda) z \frac{(\alpha z)^{i}}{i !} d U(z)\right]}
\end{gathered}
$$

and, similarly, for the case (B) we obtain

$$
\begin{gathered}
g_{\mathrm{B}}(\lambda)=\mathbf{E}_{\mathrm{B}}\left\{e^{-\lambda c_{1}}\right\} \\
=\alpha \sum_{i=0}^{\infty} \sum_{n=i+1}^{\infty} p_{n}^{(i+1) *} \mathbf{E}_{\mathrm{n}}\left\{e^{-\lambda \widehat{\tau}_{1}}\right\} \int_{0}^{\infty} e^{-(\alpha+\lambda) x} d x \int_{0}^{\infty} e^{-\lambda z} \frac{(\alpha z)^{i}}{i !} d U(z) \\
=\frac{\alpha}{\alpha+\lambda} \sum_{i=0}^{\infty} \sum_{n=i+1}^{\infty} p_{n}^{(i+1) *} \mathbf{E}_{\mathrm{n}}\left\{e^{-\lambda \widehat{\tau}_{1}}\right\} \int_{0}^{\infty} e^{-\lambda z} \frac{(\alpha z)^{i}}{i !} d U(z) .
\end{gathered}
$$

For $q_{A}(\theta)$ and $q_{B}(\theta)$, using the formula of total probability, we get the following representations for $|\theta| \leq 1$ : 


$$
\begin{gathered}
q_{\mathrm{A}}(\theta)=\alpha \sum_{i=0}^{\infty} \sum_{n=i+1}^{\infty} p_{n}^{(i+1) *} \mathbf{E}_{\mathrm{n}}\left\{\theta^{N\left(\widehat{\tau}_{1}\right)}\right\} \\
\times\left[\int_{0}^{\infty} e^{-\alpha x} d x \int_{x}^{\infty} d V(y) \int_{0}^{\infty} \frac{(\alpha(z+y-x))^{i} e^{-\alpha(z+y-x)}}{i !} d U(z)\right. \\
\left.+\frac{v(\alpha)}{\alpha} \int_{0}^{\infty} e^{-\alpha z} \frac{(\alpha z)^{i}}{i !} d U(z)\right]
\end{gathered}
$$

and

$$
q_{\mathrm{B}}(\theta)=\sum_{i=0}^{\infty} \sum_{n=i+1}^{\infty} p_{n}^{(i+1) *} \mathbf{E}_{\mathrm{n}}\left\{\theta^{N\left(\widehat{\tau}_{1}\right)}\right\} \int_{0}^{\infty} \frac{(\alpha z)^{i}}{i !} e^{-\alpha z} d U(z)
$$

The renewal-theory approach gives the following equation for $k \geq 1$ and $\gamma=A, B$ :

$$
\begin{gathered}
\mathbf{P}_{\gamma}\left\{h(t)=m, t \in c_{k}\right\}=\sum_{n=k}^{m}\left(\widehat{q} *\left(q^{\gamma}\right)^{(k-1) *}\right)_{n} \\
\times \int_{0}^{t} \mathbf{P}_{\mathrm{w}(\gamma)}\left\{h(t-y)=m, t-y \in c_{1}\right\} d\left(G_{0} * G_{\gamma}^{(k-1) *}\right)(y),
\end{gathered}
$$

where $\left(\widehat{q} *\left(q^{\gamma}\right)^{(k-1) *}\right)_{n}=\sum_{i=1}^{n} \widehat{q}_{n}\left(q_{n-i}^{\gamma}\right)^{(k-1) *}$.

Well known properties of transforms give for $\lambda>0,|\theta| \leq 1, k \geq 1$ and $\gamma=A, B$ :

$$
\begin{gathered}
\sum_{m=0}^{\infty} \theta^{m} \int_{0}^{\infty} e^{-\lambda t} \mathbf{P}_{\gamma}\left\{h(t)=m, t \in c_{k}\right\} d t= \\
=D_{\gamma}(\theta, \lambda, \alpha) g_{0}(\lambda) g_{\gamma}^{k-1}(\lambda) \widehat{q}(\theta) q_{\gamma}^{k-1}(\theta) .
\end{gathered}
$$

Of course, we also have (see (3)):

$$
\begin{gathered}
g_{0}(\lambda)=\mathbf{E}_{\text {std }}\left\{e^{-\lambda \widehat{\tau}_{0}}\right\}=1-f_{+}(1, \lambda, 0, \alpha), \\
\widehat{q}(\theta)=\mathbf{E}_{\text {std }}\left\{\theta^{N\left(\widehat{\tau}_{1}\right)}\right\}=1-f_{+}(\theta, 0,0, \alpha) .
\end{gathered}
$$

Finally, taking into consideration formulae (19) and (21) we can write the following theorem that states the PGF of LT of departure process in the system with single vacations and setup times:

Theorem 4. For any $\lambda>0,|\theta| \leq 1$ and for $\gamma=A, B$ the following formula holds true:

$$
\sum_{m=0}^{\infty} \theta^{m} \int_{0}^{\infty} e^{-\lambda t} \mathbf{P}_{\gamma}\{h(t)=m\} d t=\widehat{D}_{\mathrm{std}}(\theta, \lambda, \alpha)+\frac{D_{\gamma}(\theta, \lambda, \alpha) g_{0}(\lambda) \widehat{q}(\theta)}{1-g_{\gamma}(\lambda) q_{\gamma}(\theta)}
$$

where $\widehat{D}_{\text {std }}(\theta, \lambda, \alpha)$ and $D_{\gamma}(\theta, \lambda, \alpha)$ are found in (14) and (17), (18) respectively; $g_{0}(\lambda)$, $\widehat{q}(\theta)$ are taken from (28) and (29) respectively; and $q_{\gamma}(\theta)$ and $g_{\gamma}(\lambda)$ are derived in (22)-(25). 


\section{References}

[1] Baba Y., On the $M^{X} / G / 1$ queue with vacation, Oper. Res. Lett. 5 (1986): 93-98.

[2] Choudhury G., An $M^{X} / G / 1$ queueing system with a setup period and a vacation period, Queueing Syst. 36 (2000): 23-38.

[3] Choudhury G., A batch arrival queue with a vacation under single vacation policy, Comput. Oper. Res. 29(14) (2002): 1941-1955.

[4] Choudhury G., Kalita S., Analysis of a batch arrival Poisson queue under single vacation policy, Calcutta Stat. Assoc. Bull. 53 (2002): 81-91.

[5] Doshi N., Queueing systems with vacations - a survey, Queueing Syst. 1 (1986): $29-66$.

[6] Fuhrmann S. W., Cooper R. B., Stochastic decompositions in the $M / G / 1$ queue with generalized vacations, Operations Res. 33(5) (1985): 1117-1129.

[7] Bratiichuk M. S., Kempa W. M., Application of the superposition of renewal processes to the study of batch arrival queues, Queueing Syst. 44 (2003): 51-67.

[8] Bratiichuk M. S.; Kempa W. M., Explicit formulae for queue length of batch arrival systems, Stoch. Models 20(4) (2004): 457-472.

[9] Kempa W. M., The departure process for the queueing systems with batch arrival of customers, Stoch. Models 24(2) (2008): 246-263.

[10] Kempa W. M., GI/G/1/ $\infty$ batch arrival queueing system with a single exponential vacation, Math. Meth. Oper. Res. 69(1) (2009): 91-97.

[11] Kempa W. M., Some new results for departure process in the $M^{X} / G / 1$ queueing system with a single vacation and exhaustive service, Stoch. Anal. Appl. 28(1) (2010): 1-20.

[12] Takagi H., Queueing Analysis: A Foundation of Performance Evaluation I, Vacation and Priority Systems Part I (Elsevier, Amsterdam-New York, 1991).

[13] Tang Y., On transient departure process of $M^{X} / G / 1$ queueing system with single server vacation, J. Syst. Sci. Complex. 20(4) (2007): 562-571.

[14] Tian N., Zhang Z. G., Vacation Queueing Models: Theory and Applications (Springer, 2006). 